\title{
Quenching-Chemiluminescence Determination of Trace Amounts of L-Tyrosine Contained in Dietary Supplement by Chemiluminescence Reaction of an Iron-Phthalocyanine Complex
}

\author{
Takao Ohtomo, ${ }^{1}$ Shukuro Igarashi, ${ }^{1}$ Yoshitaka Takagai, ${ }^{2}$ and Osamu Ohno ${ }^{1}$ \\ ${ }^{1}$ Department of Biomolecular Functional Engineering, Faculty of Engineering, Ibaraki University, 4-12-1 Nakanarusawa, \\ Hitachi, Ibaraki 316-8511, Japan \\ ${ }^{2}$ Faculty of Symbiotic Systems Science, Cluster of Science and Technology, Fukushima University, Kanayagawa 1, \\ Fukushima 960-1296, Japan
}

Correspondence should be addressed to Shukuro Igarashi, igarashi@hcs.ibaraki.ac.jp

Received 18 November 2011; Revised 22 January 2012; Accepted 23 January 2012

Academic Editor: Jianxiu Du

Copyright (C) 2012 Takao Ohtomo et al. This is an open access article distributed under the Creative Commons Attribution License, which permits unrestricted use, distribution, and reproduction in any medium, provided the original work is properly cited.

\begin{abstract}
The chemiluminescence (CL) signal immediately appeared when a hydrogen peroxide solution was injected into an ironphthalocyanine tetrasulfonic acid (Fe-PTS) aqueous solution. Moreover, the CL intensity of Fe-PTS decreased by adding Ltyrosine. Based on these results, the determination of trace amounts of L-tyrosine was developed using the quenching-chemiluminescence. The calibration curve of L-tyrosine was obtained in the concentration range of $2.0 \times 10^{-7} \mathrm{M}$ to $2.0 \times 10^{-5} \mathrm{M}$. Moreover, the relative standard deviation (RSD) was $1.63 \%(n=5)$ for $2.0 \times 10^{-6} \mathrm{M}$ L-tyrosine, and its detection limits $(3 \sigma)$ were $1.81 \times 10^{-7} \mathrm{M}$. The spike and recovery experiments for L-tyrosine were performed using a soft drink. Furthermore, the determination of L-tyrosine was applied to supplements containing various kinds of amino acids. Each satisfactory relative recovery was obtained at 98 to $102 \%$.
\end{abstract}

\section{Introduction}

In vivo L-tyrosine is known as a substance with a reductive action. The L-tyrosine is a substance that generates a neurotransmitter such as adrenaline, norepinephrine (noradrenaline), or dopamine and becomes the thyroid hormone that controls the metabolism and is a precursor of melanin $[1,2]$. Therefore, the development of a method to determine small amounts of L-tyrosine is important in the medical and pharmacology fields. Moreover, the intake of L-tyrosine is also necessary for the preservation of health and is contained in nutrition supplements. Recently, the guarantee of its quality and analysis has become especially important problems in the nutritional science field.

L-tyrosine has been determined by various methods. For example, ultraviolet absorptiometry at the wavelength of $280 \mathrm{~nm}$ [3] and the Lowry method using the phenol reagent are known as a general determination analysis methods [4], but both have too low a sensitivity. Recently, many methods have been reported. They are as follows: spectrophotometry using the phenylalanine ammonia-lyase enzyme reaction [5], fluorimetry using the 1,5-bis(4,6-dichloro-1,3,5-triazinylamino) synthesis reaction [2] or using the $\mathrm{Mo}(\mathrm{VI})$-phenylfluorone quenching reaction [6], chemiluminescence (CL) using the oxidation reaction of $\mathrm{K}_{3} \mathrm{Fe}(\mathrm{CN})_{6}$ and $\mathrm{KMnO}_{4}[7-$ 9], cyclic voltammetry using the multiwalled carbon nanotube/4-amino-benzenesulfonic acid film-coated glassy carbon electrode oxidation reaction [10], UV detection of microcolumn electrophoresis [11], and HPLC-FL [12]. However, only a few rapid, simple, and highly sensitive analyses of L-tyrosine are known.

The CL method is highly sensitive, and the setup is simple and has the advantage that the measurement time is short. The CL analysis of various samples is performed in many fields such as biochemistry, clinical chemistry, and 
environmental chemistry for this reason. However, the absolute number of high sensitive and high functional CL substance is a few although the CL system using luminol or peroxyoxalate has been many reported $[13,14]$. Therefore, the appearance of a high CL substance is strongly desired. Up to now, the authors have reported about the chlorophyll CL [15] and the iron-chlorophyllin complex CL [16]. In these CLs, each compound itself has multifunctions such as a luminescence substrate, a catalyst, and an energy donor. Moreover, in the iron-chlorophyllin complex CL, the luminescence was quenched when L-ascorbic acid was made to coexist in an oxidation reaction with hydrogen peroxide. The quenching-CL determination of $10^{-8} \mathrm{M}$ for L-ascorbic acid was developed [17]. On the other hand, the CL phenomenon with respect to the iron-phthalocyanine tetrasulfonic acid (Fe-PTS) was reported $[18,19]$. The Fe-PTS complex had multi-CL functions similar to the iron-chlorophyllin complex, because the Fe-PTS as a luminescence substrate is the analog of an iron-chlorophyllin complex. In this paper, as a result of examining the Fe-PTS CL system, the coexistence of L-tyrosine significantly influenced the CL signal intensity. The quenching-CL method of L-tyrosine with Fe-PTS was then developed, especially, the determination of tyrosine in soft drink and dietary supplement were examined. The details are described as follows.

\section{Experimental}

2.1. Reagents. Iron-phthalocyanine tetrasulfonic acid was obtained from Aldrich (Milwaukee, WI, USA). Sodium tetraborate (Borax) was obtained from the Kanto Chemical Co. (Tokyo, Japan). Hydrogen peroxide was obtained from Wako Pure Chemicals Industries. (Osaka, Japan). The Ltyrosine was obtained from the Tokyo Chemical Industry Co. (Tokyo, Japan). The practical samples were obtained from a soft drink (Amino supli, Kirin beverage Co., Tokyo, Japan) and a dietary supplement (Amino body, Orihiro, Gunma, Japan). All the other reagents were of analytical grade.

2.2. Instrument. A GENELIGHT200S (the spectra range of PMT: 400-570 nm; Microtec NITI-ON, Chiba, Japan) was used for the CL measurements. The $\mathrm{pH}$ was obtained using an F-51 (HORIBA, Kyoto, Japan). The distilled water was obtained using a SWAC-500 (Shimadzu Co., Kyoto, Japan).

\subsection{Experimental Procedure}

2.3.1. Standard Procedure Concerning the Determination of L-Tyrosine. A $300 \mu \mathrm{L}$ sample of water containing L-tyrosine was injected into a glass cell using a microsyringe. A $100 \mu \mathrm{L}$ $\mathrm{pH}$ buffer solution and $50 \mu \mathrm{L}$ iron-phthalocyanine complex solution were added and mixed. Next, the cell was then placed into the cell holder of the CL detection equipment. A $50 \mu \mathrm{L}$ aliquot of hydrogen peroxide was then injected using a microsyringe and the CL signal was measured. The determination was based on the following equation. The $\Delta \mathrm{CL}=\mathrm{CL}_{0}-\mathrm{CL}_{s}$, where $\Delta \mathrm{CL}$ is the difference in the CL signal intensity, $\mathrm{CL}_{0}$ is the intensity of the $\mathrm{L}$-tyrosine additive-free

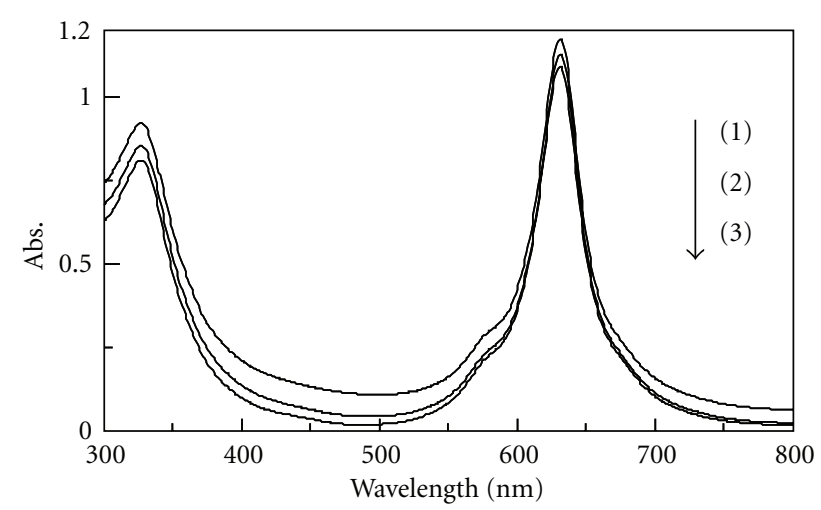

FIgURE 1: Absorption spectral change of Fe-PTS. $[\mathrm{Fe}-\mathrm{PTS}]_{T}=$ $4.0 \times 10^{-5} \mathrm{M}, \mathrm{pH}=10$, Signal: $(1)[\mathrm{L} \text {-tyrosine }]_{T}=0 \mathrm{M},(2)[\mathrm{L}-$ tyrosine $_{T}=1.0 \times 10^{-6} \mathrm{M}$, (3) $[\mathrm{L} \text {-tyrosine }]_{\mathrm{T}}=5.0 \times 10^{-5} \mathrm{M}$.

sample, and $\mathrm{CL}_{s}$ is the intensity of a certain amount of $\mathrm{L}^{-}$ tyrosine contained in the sample.

2.3.2. Spike and Recovery Experiments of L-Tyrosine in Soft Drink. A $1.0 \mathrm{~mL}$ soft drink was diluted to the marked line with distilled water in a $50 \mathrm{~mL}$ volumetric flask. Next, $5 \mathrm{~mL}$ of a $6.7 \times 10^{-6} \mathrm{M} \mathrm{L}$-tyrosine solution was added to $5 \mathrm{~mL}$ of this soft drink. The measurement was done using the standard procedure concerning the determination of $\mathrm{L}$-tyrosine.

2.3.3. The Determination of L-Tyrosine in Dietary Supplement. A $0.25 \mathrm{~g}$ sample of the tablet (the content of L-tyrosine is $6.00 \mathrm{mg}$ ) was dissolved in $15 \mathrm{~mL}$ of a $1.0 \mathrm{M}$ sodium hydroxide solution and then was diluted to the marked line with distilled water in a $250 \mathrm{~mL}$ volumetric flask. A $1.4 \mathrm{~mL}$ of this solution was diluted to the marked line with distilled water in a $10 \mathrm{~mL}$ of volumetric flask, and the CL signal was measured using the standard procedure concerning the determination of L-tyrosine.

\section{Results and Discussion}

3.1. Consideration of CL Reaction. It was examined whether L-tyrosine works as $\mathrm{H}_{2} \mathrm{O}_{2}$ scavenger. Since L-tyrosine is a reducing agent, naturally, authors think that it may work as $\mathrm{H}_{2} \mathrm{O}_{2}$ scavenger. In a previous paper [17], it is because the similar quenching-CL phenomenon by L-ascorbic acid was observed in iron-chlorophyllin/ $\mathrm{H}_{2} \mathrm{O}_{2} / \mathrm{L}$-ascorbic acid.

It was examined whether L-tyrosine works as a ligand of Fe-PTS. The absorption spectral change of Fe-PTS is shown in Figure 1. The absorbance of Fe-PTS was decreased with increasing L-tyrosine concentration. From this result, it is thought that L-tyrosine works as a ligand of Fe-PTS.

It was examined whether L-tyrosine might work as a quencher (energy transfer type). Authors performed SternVolmer plot. Stern-Volmer plot from $2.0 \times 10^{-7}$ to $2.0 \times$ $10^{-6} \mathrm{M}$ in the range of concentrations of L-tyrosine became linear. Therefore, Stern-Volmer plot showed that L-tyrosine worked as a quencher. 


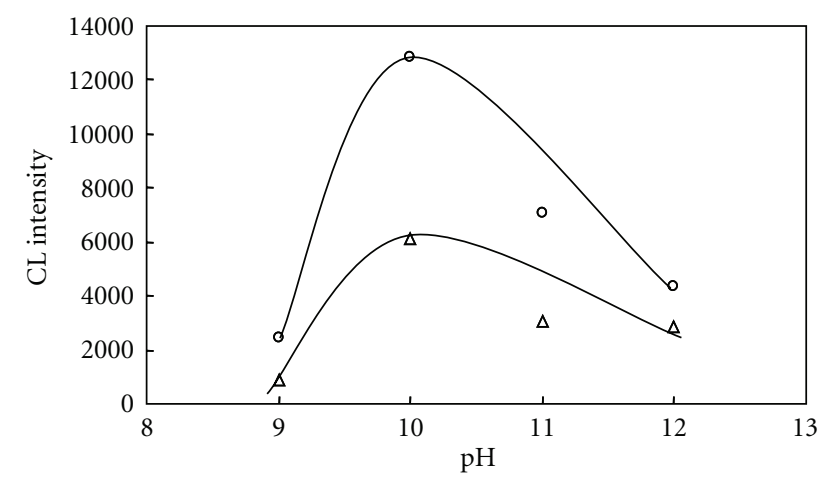

FIGURE 2: Influence of $\mathrm{pH}$ (PMT voltage: $730 \mathrm{~V})$. $[\mathrm{Fe}-\mathrm{PTS}]_{T}=4.0$ $\times 10^{-5} \mathrm{M},\left[\mathrm{H}_{2} \mathrm{O}_{2}\right]_{T}=3.2 \times 10^{-3} \mathrm{M} . \circ:[\mathrm{L}-\text { tyrosine }]_{T}=0 \mathrm{M}, \Delta:[\mathrm{L}-$ tyrosine $]_{T}=2.0 \times 10^{-6} \mathrm{M}$.

In conclusion, the function of L-tyrosine in the CL phenomenon is presumed form the above to work compositely and to happen.

3.2. Procedure Optimization. Based on the difference between the CL intensity of the blank reaction and the CL intensity for the $2.0 \times 10^{-6} \mathrm{M}$ L-tyrosine concentration that was the highest, each condition was optimized.

3.2.1. Influence of Fe-PTS Concentration. The concentration of the Fe-PTS was varied in the concentration range of $1.0 \times$ $10^{-5} \mathrm{M}$ to $5.0 \times 10^{-5} \mathrm{M}$. The $\mathrm{CL}$ intensity increased with the increasing concentration of Fe-PTS. The optimal concentration of Fe-PTS was $4.0 \times 10^{-5} \mathrm{M}$ according to the procedure optimization.

3.2.2. Influence of Hydrogen Peroxide Concentration. The concentration of the hydrogen peroxide was varied in the range of $1.6 \times 10^{-3} \mathrm{M}$ to $8.0 \times 10^{-3} \mathrm{M}$. As a result, the maximum $\mathrm{CL}_{0}$ intensity could be measured at $6.4 \times 10^{-3} \mathrm{M}$ hydrogen peroxide. However, the maximum $\triangle \mathrm{CL}$ could be obtained at $3.2 \times 10^{-3} \mathrm{M}$ hydrogen peroxide according to the procedure optimization.

3.2.3. Influence of $p H$. The relationship of the CL intensity and $\mathrm{pH}$ is shown in Figure 2. The $\mathrm{pH}$ was varied within the range of 9 to 12 . The maximum CL intensity according to the $\mathrm{pH}$ change was observed at $\mathrm{pH} 10$. The optimal $\mathrm{pH}$ is 10 according to the procedure optimization.

3.3. Calibration Curve. The L-tyrosine was measured using the CL of Fe-PTS. The CL intensity quantitatively decreased when the concentration of the coexisting L-tyrosine increased. The CL signal for various L-tyrosine concentrations is shown in Figure 3.

In the calibration curve of L-tyrosine, the relationship obtained between the concentration range of $2.0 \times 10^{-7} \mathrm{M}$ to $2.0 \times 10^{-5} \mathrm{M}$ of $\mathrm{L}$-tyrosine, and the difference in the $\mathrm{CL}$ intensity $(\Delta \mathrm{CL})$ was $y=2544 \ln (x)+39864$, where $y$ is $\Delta \mathrm{CL}$ and $x$ is the L-tyrosine concentration [M]. The correlation coefficient was 0.981 , the RSD was $1.63 \%$ ( $n=$ 5) at a $2.0 \times 10^{-6} \mathrm{M}$ L-tyrosine concentration, and the

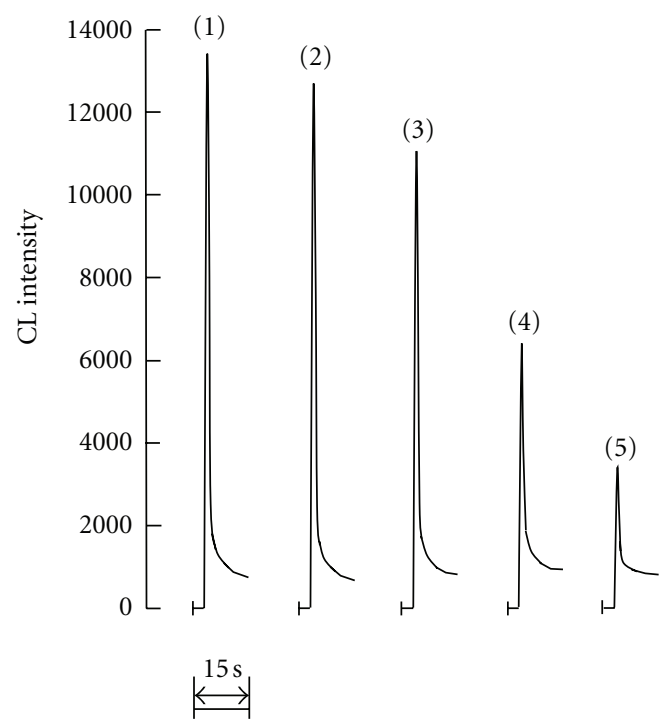

FIgURE 3: Relationship between L-tyrosine concentration and $\mathrm{CL}$ intensity (PMT voltage: $730 \mathrm{~V}$ ). $[\mathrm{Fe}-\mathrm{PTS}]_{T}=4.0 \times 10^{-5} \mathrm{M}$, $\left[\mathrm{H}_{2} \mathrm{O}_{2}\right]_{T}=3.2 \times 10^{-3} \mathrm{M}, \mathrm{pH}=10$. Curves in various concentration (M) of L-tyrosine: (1) 0 , (2) $2.0 \times 10^{-7}$, (3) $5.0 \times 10^{-7}$, (4) $2.0 \times$ $10^{-6}$, and (5) $5.0 \times 10^{-6}$.

TABLE 1: Determination for isomer of tyrosine.

\begin{tabular}{lcc}
\hline Substance & Detection limit $(\mathrm{M})$ & $\mathrm{RSD}(n=5)$ \\
\hline L-tyrosine & $1.81 \times 10^{-7} \mathrm{M}$ & $1.63 \%$ \\
D-tyrosine & $9.69 \times 10^{-8} \mathrm{M}$ & $1.99 \%$ \\
\hline
\end{tabular}

detection limit $(3 \sigma)$ was $1.81 \times 10^{-7} \mathrm{M}$. Moreover, the result of determination of tyrosine of enantiomer is shown in Table 1.

3.4. Influence of Foreign Substances. The interference by foreign substances was examined. This result is shown in Table 2. As for the allowable limit, the change in time was within $\pm 5 \%$ based on the difference in the CL intensity with the blank when no foreign substances were added. This was compared to the molar ratio with L-tyrosine. As a result, L-ascorbic acid could be present up to 5 -fold without affecting the results of the test. Also, although $\mathrm{Ni}^{2+}$ and $\mathrm{Co}^{2+}$ could be present up to equivalent without affecting the results of the test and $\mathrm{Cu}^{2+}$ could be present up to 0.5 -fold without affecting the results of the test, they could be masked up to 10-fold with the addition of EDTA as a masking reagent. Also, L-cysteine could be present up to 0.5 -fold without affecting the results of the test. It is reported that Lcysteine and $\mathrm{Zn}^{2+}$ form a complex under neutral and alkaline conditions [20]. As a result of the experiment, L-cysteine was masked up to 100 -fold with the addition of $\mathrm{Zn}^{2+}$ as a masking reagent. The proposed method can then be comparatively said to be selective as an analytical method for L-tyrosine.

3.5. Comparison with Other Analysis Methods. The proposed method and other methods were compared. The proposed method (procedure time: approximately $3 \mathrm{~min}$ ) was proved 
TABLE 2: Influence of foreign substances.

Foreign substance [Foreign substance]/[L-tyrosine]

Glutamine, methionine, serine, threonine, alanine, glycin, isoleucine, glucose, fructose, xylose, saccharose, raffinose, levulose, lactulose, galactose, nicotinic acid, nicotinamide, sodium pantothenate 1000 Asparagine, lysine, phenylalanine, histidine, leucine, proline

Tolerance limit is within $\pm 5 \%$ error for $\mathrm{CL}$ intensity when $[\mathrm{L} \text {-tyrosine }]_{T}$ is $2.0 \times 10^{-6} \mathrm{M}$.

* $[\mathrm{EDTA}]_{T}: 2.0 \times 10^{-5} \mathrm{M}$ was added as a masking reagent.

${ }^{*}\left[\mathrm{Zn}^{2+}\right]_{T}: 2.0 \times 10^{-4} \mathrm{M}$ was added as a masking reagent.

TABLE 3: Determination of L-tyrosine of dietary supplement.

\begin{tabular}{|c|c|c|c|c|}
\hline Sample & Added (M) & Found (M) & Recovery (\%) & $\operatorname{RSD}(n=5)$ \\
\hline Sample $A^{\text {a }}$ (soft drink) & $2.00 \times 10^{-6} \mathrm{M}$ & $1.96 \times 10^{-6} \mathrm{M}$ & $98 \%$ & $2.42 \%$ \\
\hline $\begin{array}{l}\text { Sample } \mathrm{B}^{\mathrm{b}} \\
\text { (component indicated value of supplement)* }\end{array}$ & - & $\begin{array}{c}2.04 \times 10^{-6} \mathrm{M} \\
\left(2.00 \times 10^{-6} \mathrm{M}\right)^{*}\end{array}$ & - & $2.95 \%$ \\
\hline
\end{tabular}

${ }^{a}$ Component: aspartic acid, arginine, lysine, glutamic acid, glycine, proline, alanine, leucine, isoleucine, valine, ornithine, citrulline.

${ }^{\mathrm{b}}$ Component: protein, lipid, carbohydrate, sodium, vitamin $\mathrm{B}_{1}$, vitamin $\mathrm{B}_{6}$, twenty kinds of the amino acid including the $\mathrm{L}$-tyrosine.

* Indicated value of medicine.

to be rapid and simple, but the detection limits (detection limits: $1.81 \times 10^{-7} \mathrm{M}$ ) are poor for fluorophotometry by the synthesis reaction with 1,5-bis(4,6-dichloro-1,3,5triazinyl-amino) (detection limits: $6.8 \times 10^{-8} \mathrm{M}$, procedure time: $30 \mathrm{~min}$ ) [2]. Also, the proposed method was rapid, simple, and highly sensitive compared to spectrophotometry using the phenylalanine ammonia-lyase enzyme reaction (detection limits: $5.0 \times 10^{-6} \mathrm{M}$, procedure time: $2 \mathrm{~h}$ ) [5] and quenching-fluorophotometry using $\mathrm{Mo}(\mathrm{VI})$-phenyl-fluorone (detection limits: $5.2 \times 10^{-7} \mathrm{M}$, procedure time: $5 \min )$ [6].

3.6. The Determination of the L-Tyrosine in Dietary Supplement. The determination of L-tyrosine in dietary supplement is shown in Table 3.

The L-tyrosine in a soft drink was determined. The determined concentration of L-tyrosine was $1.96 \times 10^{-6} \mathrm{M}$ for $2.00 \times 10^{-6} \mathrm{M}$, and the recovery was $98 \%$. Moreover, the L-tyrosine in medicine (supplement) was determined. The indicated value of the components in the supplement was measured by the Japan Food Research Laboratories, and the various amino acids were measured by the amino acid analytical method using ninhydrin reaction. The experimental value that had been obtained by the measurement was $2.04 \times$ $10^{-6} \mathrm{M}$ for the tabulated one of $2.00 \times 10^{-6} \mathrm{M} \mathrm{L}$-tyrosine. Satisfactory results for each sample were obtained using this method.

\section{Conclusion}

In this study, rapid, simple, and highly sensitive analysis method of trace amounts of L-tyrosine based on the CL reaction of Fe-PTS was developed. In the future, a new FIA
CL-detection will be developed for application to multisamples containing trace amounts of L-tyrosine.

\section{References}

[1] A. J. Gelenberg, C. J. Gibson, and J. D. Wojcik, "Neurotransmitter precursors for the treatment of depression," Psychopharmacology Bulletin, vol. 18, no. 1, pp. 7-18, 1982.

[2] W. Wei, W. Hong-Jian, J. Chong-Qiu, and S. Jing-Min, "Spectrofluorimetric Determination of Trace Amounts of Tyrosine with 1,5-Bis(4,6-dicholro-1,3,5-triazinylamino) naphthalene," Chinese Journal of Analytical Chemistry, vol. 35, no. 12, pp. 1772-1775, 2007.

[3] O. Warburg and W. Christian, "Isolierung und Kristallisation des Garungsferments enolase," Biochemische Zeitschrift, vol. 310, pp. 384-421, 1941.

[4] O. H. Lowry, N. J. Rosebrough, A. L. Farr, and R. J. Randall, "Protein measurement with the Folin phenol reagent," The Journal of Biological Chemistry, vol. 193, no. 1, pp. 265-275, 1951.

[5] F. Wibrand, "A microplate-based enzymatic assay for the simultaneous determination of phenylalanine and tyrosine in serum," Clinica Chimica Acta, vol. 347, no. 1-2, pp. 89-96, 2004.

[6] X. Zhu and S. Xu, "Determination of l-tyrosine by $\beta$-cyclodextrin sensitized fluorescence quenching method," Spectrochimica Acta. Part A, vol. 77, no. 3, pp. 566-571, 2010.

[7] M. C. Sanfeliu Alonso, L. Lahuerta Zamora, and J. Martínez Calatayud, "Determination of tyrosine through a FIA-direct chemiluminescence procedure," Talanta, vol. 60, no. 2-3, pp. 369-376, 2003.

[8] J. W. Costin, P. S. Francis, and S. W. Lewis, "Selective determination of amino acids using flow injection analysis coupled with chemiluminescence detection," Analytica Chimica Acta, vol. 480, no. 1, pp. 67-77, 2003.

[9] P. S. Francis, C. M. Hindson, J. M. Terry et al., "Enhanced 
permanganate chemiluminescence," Analyst, vol. 136, no. 1, pp. 64-66, 2011.

[10] K. J. Huang, D. F. Luo, W. Z. Xie, and Y. S. Yu, "Sensitive voltammetric determination of tyrosine using multi-walled carbon nanotubes/4-aminobenzeresulfonic acid film-coated glassy carbon electrode," Colloids and Surfaces B, vol. 61, no. 2, pp. 176-181, 2008.

[11] L. Li, Y. Z. He, W. E. Gan, X. K. Wang, H. Y. Xie, and Y. Gao, "Electrophoretic separation with 2-mm inner diameter fusedsilica microcolumn packed with quartz microncrystals and its application," Talanta, vol. 79, no. 2, pp. 460-465, 2009.

[12] R. Kand'ár and P. Zakova, "Determination of phenylalanine and tyrosine in plasma and dried blood samples using HPLC with fluorescence detection," Journal of Chromatography B, vol. 877, no. 30, pp. 3926-3929, 2009.

[13] G. E. Colllns and S. L. Rose-Pehrsson, "Chemiluminescent chemical sensors for oxygen and nitrogen dioxide," Analytical Chemistry, vol. 67, no. 13, pp. 2224-2230, 1995.

[14] T. Jonsson and K. Irgum, "Very fast peroxyoxalate chemiluminescence," Analytica Chimica Acta, vol. 400, no. 1-3, pp. 257264, 1999.

[15] T. Nagoshi and S. Igarashi, "Chlorophyll chemiluminescence emitted by chlorin ring cleavage with acetonitrile and hydrogen peroxide," Chemistry Letters, vol. 34, no. 1, pp. 22-23, 2005.

[16] T. Nagoshi, O. Ohno, T. Kotake, and S. Igarashi, "Chemiluminescence of iron-chlorophyllin," Luminescence, vol. 20, no. 6, pp. 401-404, 2005.

[17] T. Kato, O. Ohno, T. Nagoshi, Y. Ichinose, and S. Igarashi, "Determination of small amounts of L-ascorbic acid using the chemiluminescence of an iron-chlorophyllin complex," Analytical Sciences, vol. 21, no. 5, pp. 579-581, 2005.

[18] T. Ohtomo and S. Igarashi, "Functions of iron-phthalocyanine complex in the porphyrin analogue chemiluminescence," in Proceedings of the International Symposium on Catalysis and Fine Chemicals, p. 206, Seoul, Republic of Korea, December, 2009.

[19] S. Igarashi, Y. Tanaka, and T. Kuriki, "Measuring method of new chemiluminescence reagent, new chemiluminescence method, and substance detected that uses it and kits used for it," Japanese Patent Publication, March 24, 2011-057818, 2011.

[20] A. Sato, Y. Tanimoto, and T. Imamura, "Formation, chemical structure, and preparation of Zinc-Cysteine complex," Journal of the Faculty of Applied Biological Science, vol. 23, pp. 1-7, 1984. 


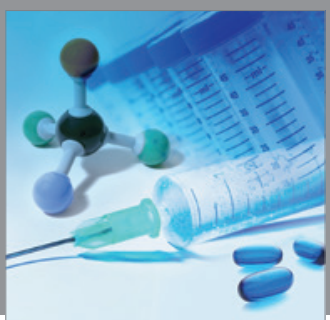

International Journal of

Medicinal Chemistry

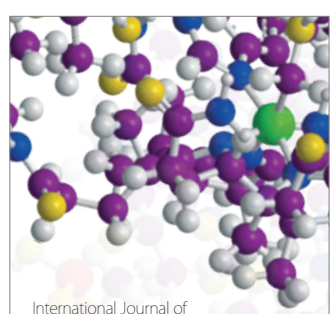

Carbohydrate Chemistry

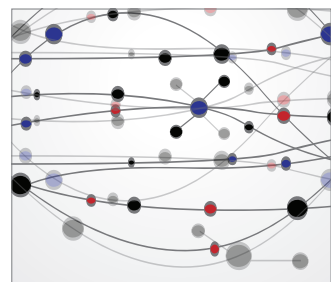

The Scientific World Journal
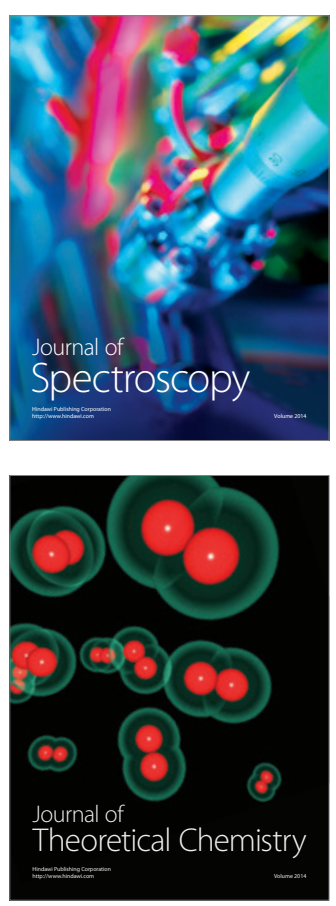
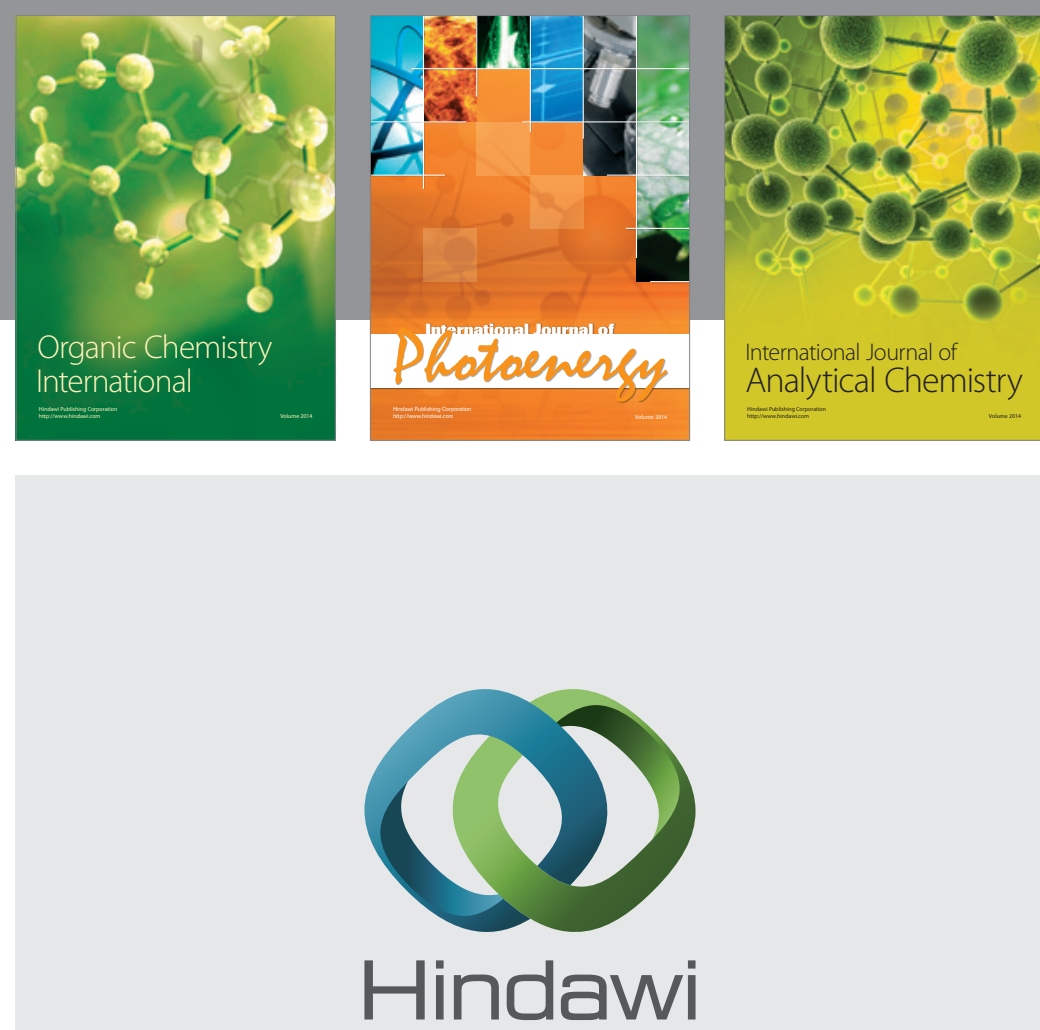

Submit your manuscripts at

http://www.hindawi.com
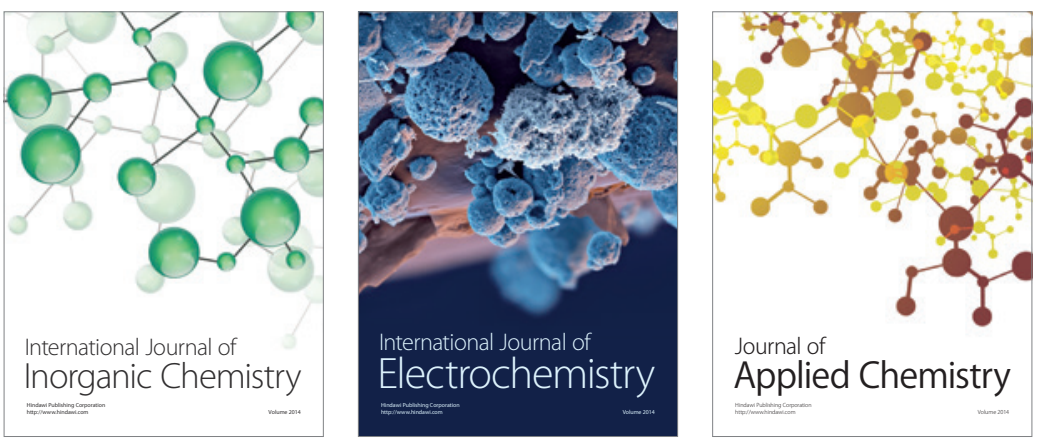

Journal of

Applied Chemistry
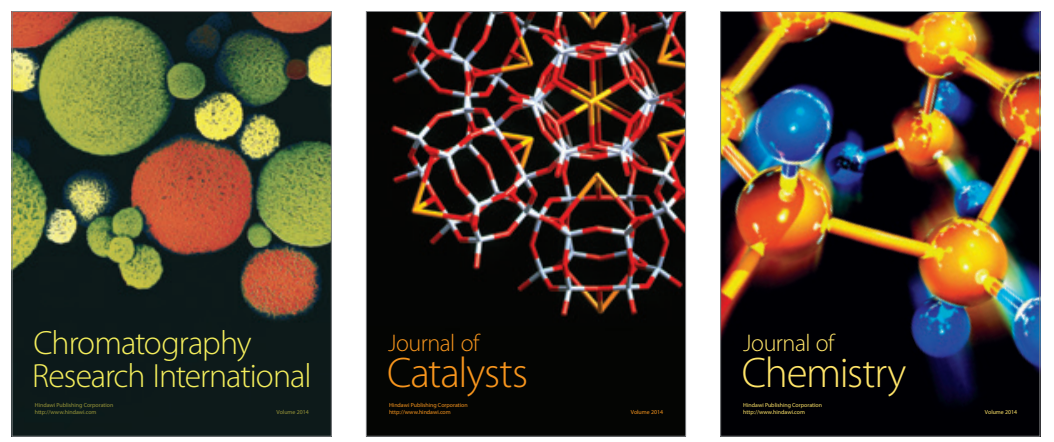
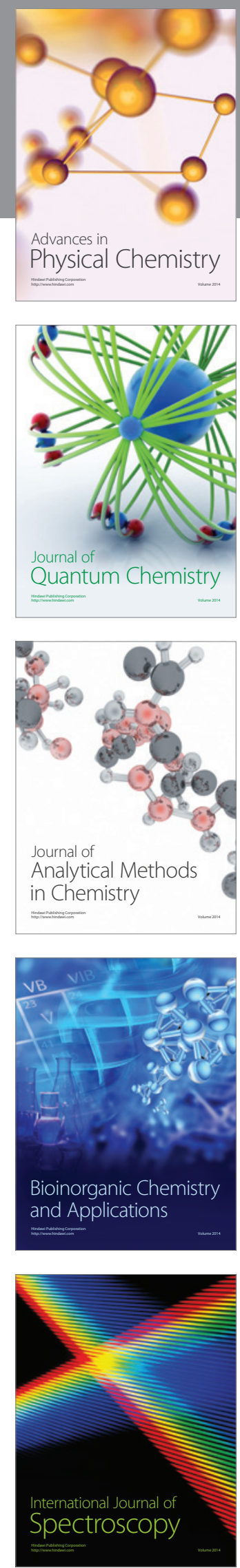